\title{
Peroxisomal Membrane Contact Sites in Mammalian Cells
}

\author{
Chao Chen ${ }^{1+}$, Jing $\mathrm{Li}^{2+}$, Xuhui Qin ${ }^{3 \dagger}$ and Wei Wang ${ }^{3 *}$ \\ ${ }^{1}$ Department of Orthopaedics, Union Hospital, Tongji Medical College, Huazhong University of Science and Technology, \\ Wuhan, China, ${ }^{2}$ Department of Integrated Traditional Chinese and Western Medicine, Tongji Hospital, Tongji Medical \\ College, Huazhong University of Science and Technology, Wuhan, China, ${ }^{3}$ Department of Human Anatomy, School of Basic \\ Medicine, Tongji Medical College, Huazhong University of Science and Technology, Wuhan, China
}

\section{OPEN ACCESS}

\author{
Edited by: \\ Laura Lackner, \\ Northwestern University, \\ United States
}

Reviewed by:

Michael Schrader,

University of Exeter, United Kingdom

Markus Islinger,

Heidelberg University, Germany

Marc Fransen

KU Leuven, Belgium

${ }^{*}$ Correspondence:

Wei Wang

wekle@hust.edu.cn

${ }^{\dagger}$ These authors have contributed equally to this work

Specialty section:

This article was submitted to

Molecular Medicine,

a section of the journal

Frontiers in Cell and Developmental

Biology

Received: 26 January 2020

Accepted: 28 May 2020

Published: 23 June 2020

Citation:

Chen C, Li J, Qin X and Wang W (2020) Peroxisomal Membrane

Contact Sites in Mammalian Cells.

Front. Cell Dev. Biol. 8:512.

doi: 10.3389/fcell.2020.00512
Peroxisomes participate in essential cellular metabolic processes, such as oxidation of fatty acids (FAs) and maintenance of reactive oxygen species (ROS) homeostasis. Peroxisomes must communicate with surrounding organelles to exchange information and metabolites. The formation of membrane contact sites (MCSs), where proteinprotein or protein-lipid complexes tether the opposing membranes of two organelles, represents an essential means of organelle crosstalk. Peroxisomal MCS (PO-MCS) studies are emerging but are still in the early stages. In this review, we summarize the identified PO-MCSs with the ER, mitochondria, lipid droplets, and lysosomes in mammalian cells and discuss their tethering mechanisms and physiological roles. We also highlight several features of PO-MCSs that may help future studies.

Keywords: peroxisomes, membrane contact sites, tethering complexes, metabolism, organelle crosstalk

Peroxisomes are essential single-membrane-bound organelles present in virtually all eukaryotic cells (Smith and Aitchison, 2013). Peroxisomes play indispensable roles in both catabolic (degradative) and anabolic (biosynthetic) metabolism through the enzymes located in the peroxisomal matrix (Waterham et al., 2016). The primary catabolic pathways include the oxidation of fatty acids (FAs) and the detoxification of glyoxylates. The main anabolic pathways include the biosynthesis of bile acids, ether phospholipids, and docosahexaenoic acids. In addition, peroxisomes are involved in the production and decomposition of reactive oxygen species (ROS), which is partly coupled to the above peroxisomal metabolic pathways. Peroxisomes can serve as signaling platforms for antiviral innate immunity (Cook et al., 2019), and ROS modulated mTORC1 activity (Zhang et al., 2013, 2015).

Peroxisome deficiency causes severe human diseases, emphasizing the crucial role of peroxisomes. The diseases fall into two main categories: single peroxisomal enzyme deficiencies and peroxisome biogenesis disorders (PBDs) (Waterham et al., 2016). PBDs are inherited in an autosomal recessive manner and affect the overall assembly and function of peroxisomes. Patients with Zellweger syndrome (ZS), the most severe form of PBDs, fail to make any developmental progress and die at an early age (Suzuki et al., 2001). Depending on the organism, tissue, cell type, and environmental factors, the functions of peroxisomes can change to meet the metabolic needs of cells (Mast et al., 2020).

Organelles are cellular compartments that perform specific enzymatic reactions. However, organelles cannot exert their activities alone and must exchange information and metabolites with others to coordinate cellular functions. Several mechanisms have been proposed for this crosstalk, including vesicular trafficking and signal transduction pathways (Shai et al., 2016). Accumulating studies now show that membrane contact sites (MCSs) represent a fast and efficient way to 
communicate between organelles (Prinz et al., 2020). A bona fide MCS is defined as follows: (1) close apposition $(10 \sim 30 \mathrm{~nm})$ of the membranes from two tethered organelles; (2) lack of membrane fusion or transient hemifusion may occur; (3) enrichment of specific proteins and/or lipids at the MCS; and (4) regulation of the composition and/or function of one or two of the organelles (Prinz, 2014).

For many years, electron microscopy (EM) studies in fungi, plants, and mammals revealed that peroxisomal membranes are juxtaposed to many other organelles, such as the ER, lipid droplets (LDs), plasma membrane (PM), mitochondria, and chloroplasts (Schrader et al., 2013). The simultaneous fluorescent labeling of six organelles in COS-7 cells reveals that peroxisomes associate with the ER, mitochondria, LDs, the Golgi, and lysosomes (Valm et al., 2017). To date, the tethering complexes and physiological roles of peroxisomal MCSs (PO-MCSs) with the ER, mitochondria, LDs, and lysosomes have been investigated in mammalian cells (Castro et al., 2018; Sargsyan and Thoms, 2020; Schrader et al., 2020). In this review, we focus on these identified PO-MCSs in mammalian cells and discuss several features of PO-MCSs.

\section{PEROXISOME-ER-MCSS}

In human cells, the biosynthesis of unsaturated FAs, sterols, ether phospholipids, and bile acids involves both the ER and peroxisomes and requires intimate crosstalk. Metabolic defects in these organelles lead to severe human diseases (Schrader et al., 2013). The ER represents the largest membrane-bound organelle in eukaryotic cells and forms MCSs with many other organelles, including peroxisomes. Although peroxisome-ER (PO-ER) associations were identified by EM in mammalian cells nearly 50 years ago (Novikoff and Novikoff, 1972), the MCSs mediating their crosstalk remained elusive.

Acyl-CoA binding domain containing 5 (ACBD5) is a peroxisomal tail-anchored membrane protein, and its deficiency can cause a defect in peroxisomal very-long-chain FA metabolism (Ferdinandusse et al., 2017). ACBD5 and its fungal ortholog, ATG37, have been suggested to play critical roles in phagophore formation during pexophagy (Nazarko et al., 2014). VAPA and VAPB (VAPA/B) are tail-anchored and ER vesicle-associated membrane proteins. These proteins can act as the MCS tether through the major sperm protein (MSP) domain that interacts with the two phenylalanines in an acidic tract (FFAT) motif of client proteins localized in the opposing organelles (Lev et al., 2008). Two independent studies showed that the tether ACBD5VAPA/B mediates the formation of PO-ER-MCS (Costello et al., 2017a; Hua et al., 2017; Figure 1A).

In these studies, super-resolution microscopy and EM visualization revealed that the two organelles were in proximity to each other. Cooverexpression of ACBD5 and VAPA/B or their knockdowns increases and decreases MCS formation, respectively, suggesting that tethering relies on the ACBD5VAPA/B interaction. As shown for other VAPA/B interacting proteins, ACBD5 interacts with the MSP domain of VAPA/B through the FFAT motif. ACBD4, a second ACBD family protein,

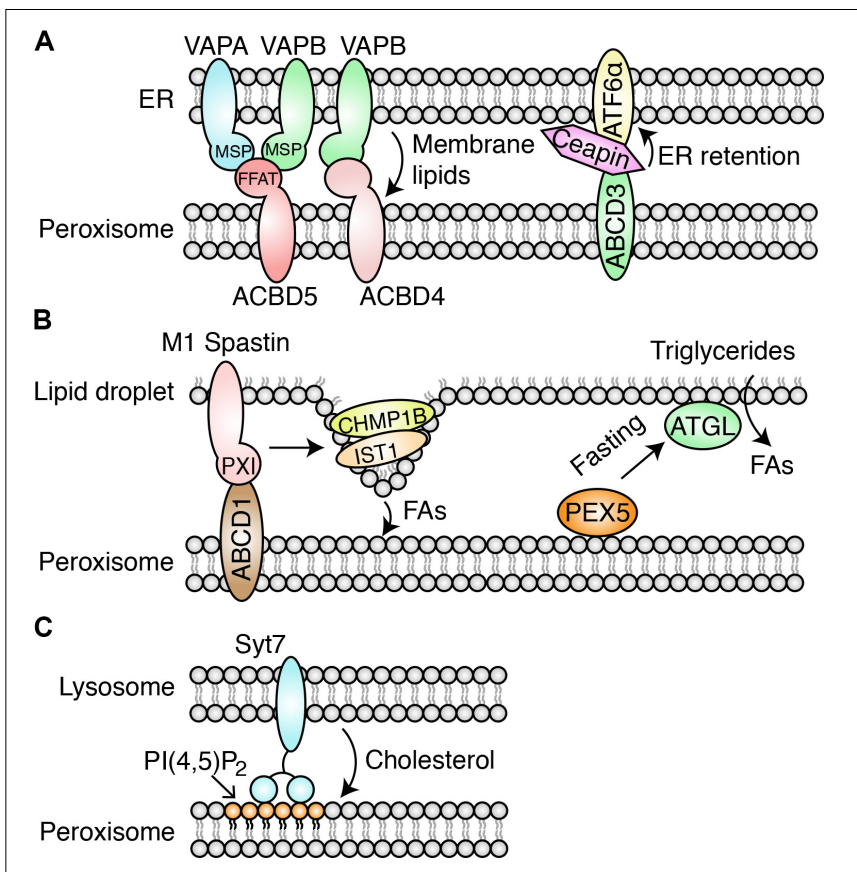

FIGURE 1 | Identified PO-MCSs in mammalian cells. (A) PO-ER-MCS. At the MCS, the FFAT motif in ACBD5 interacts with the MSP domain in VAPA/B. The ACBD4/5-VAPA/B-tethered MCS facilitates lipid transfer from the ER to peroxisomes and promotes the expansion of peroxisomal membranes. Ceapin sequesters ATF6 $\alpha$ at the ER by tethering ATF6 $\alpha$ to peroxisomal ABCD3. (B) PO-LD-MCS. M1 Spastin interacts with ABCD1 through the peroxisome-interacting (PXI) region. M1 Spastin recruits the ESCRT-III subunits IST1 and CHMP1B to the surface of LDs to promote FA trafficking through the MCS. ATGL is recruited to the MCS by PEX5 in response to fasting stress, promoting lipolysis of triglycerides stored in LDs.

(C) PO-Lyso-MCS mediated by $\mathrm{PI}(4,5) \mathrm{P}_{2}$ and Syt7. This MCS facilitates cholesterol trafficking from lysosomes to peroxisomes.

also interacts with VAPB for PO-ER associations (Costello et al., 2017b; Figure 1A).

Peroxisomes can proliferate through the growth and division model, which involves membrane elongation, constriction, and fission (Schrader et al., 2012). Since peroxisomes share division proteins with mitochondria (e.g., DRP1, MFF, and FIS1) (Smith and Aitchison, 2013) and ER-MCSs have been shown to mark mitochondrial or endosomal fission sites and modulate their division (Friedman et al., 2011; Rowland et al., 2014), it is reasonable to hypothesize that PO-ER-MCSs play a role in peroxisome division. However, peroxisome biogenesis is unaffected in ACBD5-deficient cells (Ferdinandusse et al., 2017; Yagita et al., 2017), and peroxisome morphology is normal in cells with reduced PO-ER-MCSs due to ACBD5/VAP silencing (Costello et al., 2017a; Hua et al., 2017). These results suggest that the ACBD5-VAPA/B tethered MCSs may not play a role in peroxisome division.

The phospholipid contents of peroxisome membranes are rich in phosphatidylcholine (PC) and phosphatidylethanolamine (PE), similar to the contents of the ER (Hardeman et al., 1990). The peroxisome is unable to generate membrane lipids locally because it does not contain biosynthesis enzymes. MFF 
and DRP1 are two crucial molecules involved in peroxisome and mitochondrion fission, and their deficiencies result in pronounced elongation of the two organelles, likely through a constant transfer of lipids from the ER to peroxisomes (Schrader et al., 2012). It has been found that disrupting the ACBD5-VAPA/B tethered MCS in MFF- or DRP1-deficient cells significantly inhibited the elongation of peroxisomes but not of mitochondria, suggesting that the MCS may supply the lipids required for peroxisome membrane expansion.

Both groups found that disrupting the ACBD5-VAPA/B tethered MCS increases the movement of peroxisomes, suggesting that the MCS negatively regulates peroxisome motility through ER anchoring. A motility-restricting role is also indicated for the yeast PO-ER-MCS, which can retain a fraction of peroxisomes by anchoring with the ER in mother cells when peroxisomes passage into the daughter cells (Knoblach et al., 2013). In addition, Hua et al. (2017) found a potential role of PO-ER-MCSs in the synthesis of plasmalogen phospholipids and the maintenance of cellular cholesterol levels. VAP proteins also play roles in lipid transfer at ER-MCSs with other organelles, such as the Golgi, mitochondria, and LDs (Kamemura and Chihara, 2019). Expression of an artificial PO-ER tether without any functional domain partially restored peroxisomal membrane expansion after ACBD5 silencing (Costello et al., 2017a), suggesting that VAPA/B and ACBD5 do not play an active lipidtransferring role in PO-ER-MCSs. Deficiency in both the ACBD5 and VAPB genes has been implicated in human disorders, emphasizing the significance of the identified PO-ER-MCSs (Kim et al., 2010; Abu-Safieh et al., 2013; Ferdinandusse et al., 2017; Yagita et al., 2017).

Other PO-ER-MCSs have also been identified. The MCS tethered by the peroxisomal $\mathrm{PI}(4,5) \mathrm{P} 2$ and ER-resident extended synaptotagmins (E-Syts) may facilitate the transport of cholesterol from the peroxisome to the downstream ER organelle (Xiao et al., 2019). ATF6 $\alpha$ is an unfolded protein response (UPR) sensor, which traffics to the Golgi apparatus for proteolysis and its subsequent movement to the nucleus for transcriptional activation during ER stress (Haze et al., 1999). A genome-wide CRISPR interference screening revealed that the drug Ceapin induces PO-ER-MCS formation by forcing the interaction between ATF6 $\alpha$ and the peroxisomal transmembrane protein ABCD3 (Torres et al., 2019). The ATF6 $\alpha$-ABCD3 interaction sequesters ATF6 $\alpha$ at the ER and inhibits its activity as the UPR sensor (Figure 1A). Notably, the MCS does not require the known $\mathrm{ACBD} 4 / 5-\mathrm{VAPA} / \mathrm{B}$ tether. The study suggests that Ceapin may be used for the treatment of cancer, since cancer development relies on active ATF6 $\alpha$ signaling; this finding presents a novel drug-development strategy for targeting the MCS.

\section{PEROXISOME-MITOCHONDRION-MCSS}

Peroxisomes and mitochondria must communicate with each other to meet the metabolic needs of cells. The best examples are illustrated by the $\beta$-oxidation of FAs and metabolism of ROS (discussed below). These processes share some crucial division proteins (Delille et al., 2009), de novo biogenesis of peroxisomes relies on mitochondrion-derived vesicles (Sugiura et al., 2017), and these organelles function cooperatively in antiviral signaling and defense (Kagan, 2012). In addition, defective mitochondria are observed in several peroxisomal disorders (Baumgart et al., 2001; Lopez-Erauskin et al., 2013).

In the 1970s, a close spatial peroxisome-mitochondrion (POMito) association was observed in the myocardium of rodents and primates by a peroxidative catalase activity-based alkaline DAB staining method (Hicks and Fahimi, 1977). Overexpression of PEX11 $\beta$, a critical peroxisome division factor, induces the formation of mitochondrion-interacting membrane protrusions (Kustatscher et al., 2019). The PO-Mito interaction increases when cells are infected with an RNA virus, suggesting a critical role of PO-Mito-MCSs in RNA virus infections (Horner et al., 2011). The role of PO-Mito-MCSs has been implicated in the transfer of metabolites involved in steroid biosynthesis in mouse Leydig tumor cells (Fan et al., 2016). This study suggests that the tethering complex consists of a splice isoform of an acyl-CoA binding domain-containing protein, enoyl-CoA $\delta$ isomerase 2 (ACBD2/ECI2). However, as ACBD2/ECI2 proteins are transported into the matrix of both mitochondria and peroxisomes, their tethering role needs further investigation.

Both peroxisomes and mitochondria possess separate $\beta$-oxidation pathways in mammalian cells, which are different from those in yeast and plants, whose peroxisomes are the sole organelles for $\beta$-oxidation of FAs. The enzymatic steps of $\beta$-oxidation of FAs in these two organelles are similar but differ in the catalyzed substrates. For example, peroxisomes preferentially catalyze the $\beta$-oxidation of very-long-chain FAs, while the mitochondrial $\beta$-oxidation system catalyzes medium and long-chain FAs. The chain-shortened FAs, as well as the acetyl-CoA generated through peroxisomal $\beta$-oxidation, need to be directed to mitochondria for further oxidation and ATP production. Different mechanisms have been proposed for the transfer of these metabolites, including the carnitine system, membrane pores, and vesicular transport (Antonenkov and Hiltunen, 2012; Sugiura et al., 2014). A high content screening approach for PO-MCSs in yeast suggests that the tethering complex (Pex11, Fzo1, and Pex34) contributes to PO-Mito-MCS formation, which facilitates the transfer of $\beta$-oxidation products (Shai et al., 2018). The conserved function of mammalian PO-Mito-MCS remains to be determined.

In addition, both organelles contain pro-oxidant and antioxidant systems. Increasing evidence demonstrates that these organelles undergo intimate crosstalk during cellular redox metabolism (Fransen and Lismont, 2018). For example, inhibition of the peroxisomal antioxidant enzyme catalase or excess ROS generation in peroxisomes disrupts mitochondrial redox (Walton and Pizzitelli, 2012; Wang et al., 2013). Expression of peroxisome-targeted PRDX5 (Peroxiredoxin-5) protects cells from the oxidative insults generated from mitochondria (Walbrecq et al., 2015). The ER-Mito-MCS has been shown to transfer ER-derived ROS to mitochondria, causing the cell to be sensitive to mitochondrial apoptosis (Verfaillie et al., 2012). ER-Mito-MCS-mediated $\mathrm{Ca}^{2+}$ transfer stimulates ROS translocation from mitochondria to the MCS. The local $\mathrm{H}_{2} \mathrm{O}_{2}$ 
enrichment at the MCS further augments the ER-Mito $\mathrm{Ca}^{+}$ flux, likely through $\mathrm{H}_{2} \mathrm{O}_{2}$-mediated oxidation of specific thiol groups of the pump receptor IP3 (Booth et al., 2016). To date, no studies have shown the role of PO-Mito-MCS in redox crosstalk. It is tempting to speculate that such a PO-Mito-MCS exists for maintaining cellular redox balance.

The above results suggest that MCSs play critical roles in PO-Mito crosstalk. However, the mechanism of POMito-MCSs remains elusive. Future studies identifying the tethering components would increase our understanding of POMito crosstalk.

\section{PEROXISOME-LIPID DROPLET-MCSS}

Lipid droplets are essential and significant lipid storage organelles for neutral lipids, such as triacylglycerol and cholesterol ester. The lipids must be imported into oxidative organelles, such as mitochondria and peroxisomes, for $\beta$-oxidation to enable cellular homeostasis (Thiam and Dugail, 2019). Indeed, aberrant FA metabolism in LDs is implicated in severe physiological disorders, including lipodystrophy and neurological diseases (Welte, 2015). Defective FA metabolism in peroxisomes causes an accumulation of LDs, as found in patients with peroxisomal disorders (Schaumburg et al., 1972; Engelen et al., 2012). Changes in the number and size of LDs are observed in peroxisomedeficient mice (Baes et al., 1997; Dirkx et al., 2005). These results suggest that the two organelles need to cooperate to maintain cellular homeostasis. Two recent studies find that they communicate through the peroxisome-lipid droplet (PO-LD)MCSs as follows (Chang et al., 2019; Kong et al., 2020).

Spastin is a microtubule-severing protein that belongs to the AAA (ATPases Associated with various cellular Activities) family. The longer M1 variant (M1 Spastin) encodes an N-terminal hydrophobic hairpin motif, which targets M1 Spastin to LDs (Errico et al., 2002). Chang et al. (2019) demonstrated that M1 Spastin forms a tethering complex with the peroxisome membrane protein ABCD1 to maintain the PO-LD MCS for FA trafficking from LDs to peroxisomes. M1 Spastin also recruits the curvature-generating ESCRT III subunits IST1 and CHMP1B to the surface of LDs to promote FA trafficking (Figure 1B). The trafficking of FAs through the MCS prevents the accumulation of peroxidated lipids following oxidative stress (Chang et al., 2019). Spastin gene mutations are the most common cause of hereditary spastic paraplegias (HSPs), a group of inherited neurological disorders (Blackstone, 2018). The results suggest that failure to induce LD-PO-MCS formation and subsequent FA transport by the M1 Spastin mutation (K388R) may contribute to HSP (Chang et al., 2019), highlighting the critical role of PO-LD-MCS in HSP pathogenesis.

Another study revealed the role of PO-LD-MCS in regulating lipolysis, the hydrolysis of lipid metabolites stored in LDs (Kong et al., 2020). During fasting, the increase in PO-LDMCSs facilitates the spatial translocation of adipose triglyceride lipase (ATGL) onto LDs for lipolysis. PEX5 recruits ATGL to the MCS, independent of its role as the receptor for the import of peroxisomal matrix proteins (Figure 1B). It would be interesting to investigate whether the M1 Spastin-ABCD1 complex tethers this MCS for lipolysis. Importantly, these results are verified by studies in multiple models, such as mammalian adipocytes, Caenorhabditis elegans, and mice. The study reveals the physiological significance of the PO-LD-MCS in maintaining energy homeostasis in response to nutritional status. As aberrant lipolysis is associated with severe metabolic diseases, such as obesity and diabetes, this study suggests PO-LD-MCSs as a potential therapeutic target for these diseases.

As discussed above, the MCS can facilitate the transport of lipids from LDs to peroxisomes for their breakdown or function as a station enriched with lipases to hydrolyze the lipids in LDs. Detection of peroxisome-derived metabolites (e.g., etherlinked lipids) in LDs suggests that they may be transferred from peroxisomes to LDs through the MCS (Bartz et al., 2007). The bidirectional transfer at the PO-LD-MCS awaits discovery.

\section{PEROXISOME-LYSOSOME-MCSS}

Lysosomes are degradative and metabolic organelles. Many essential metabolic processes, such as those for cholesterol, occur in this organelle. Cholesterol is a crucial component for maintaining the fluidity, permeability, and organization of mammalian membranes. Cholesterol is unevenly distributed in cellular membrane structures, with the highest contents (60 80\% of total cellular cholesterol) in the PM (Lange et al., 1989). The low-density lipoprotein-derived cholesteryl ester is internalized through endocytosis, hydrolyzed to unesterified cholesterol in lysosomes and further delivered to downstream organelles (Chang et al., 2006).

A genome-wide pooled shRNA screen identified a list of peroxisome genes required for cholesterol trafficking (Chu et al., 2015). These genes are either essential for peroxisome biogenesis or involved in its metabolism. These authors observed a dramatic accumulation of cholesterol in lysosomes and a significant reduction of peroxisome-lysosome (PO-Lyso)-MCS formation in cells deficient in these peroxisome genes. These authors further revealed that peroxisomal $\mathrm{PI}(4,5) \mathrm{P}_{2}$ lipid and lysosomal synaptotagmin VII (Syt7) protein tether the MCS for cholesterol trafficking from lysosome to peroxisome (Chu et al., 2015; Figure 1C). Time-lapse visualization reveals that the POLyso-MCS is transient. Depletion and repletion of cholesterol inhibit and recover the MCS, respectively, indicating that MCS formation depends on the presence of cholesterol. The followup study found that PIP4K2A, a PI(5)P-kinase, contributes to the generation of peroxisomal PI(4,5)P2, enhances PO-Lyso-MCS formation, and promotes cholesterol trafficking (Hu et al., 2018).

Drastic amounts of cholesterol accumulate in the mouse model and the patient fibroblasts with peroxisomal disorders, as these authors have examined. This study suggests that part of the pathological mechanisms of peroxisomal disorders may be attributed to the blockage of cholesterol trafficking caused by defective PO-Lyso-MCS formation and may provide novel strategies for their diagnoses and treatments (Chu et al., 2015). However, as the experimental method for peroxisome purification in this study is under debate (Schrader et al., 2020) 
and the study does not examine the loss of PO-Lyso-MCS in these patient fibroblasts, other mechanisms may contribute to abnormal cholesterol accumulation. Studies have shown that the initial step of plasmalogen synthesis occurs in peroxisomes and that its deficiency interferes with the transport of cholesterol from the PM or endocytic compartments to the ER (Thai et al., 2001; Munn et al., 2003). The accumulation of lysosomal cholesterol in peroxisome-deficient cells could result from insufficient plasmalogen synthesis due to loss of peroxisome functions.

In addition, lysosomes can serve as the signaling hub for the vital growth regulator mTOR. When there are sufficient nutrients, mTOR is recruited to lysosomes and activated by lysosomal Rheb proteins to promote downstream anabolism for cellular growth (Saxton and Sabatini, 2017). Interestingly, a study showed that peroxisome-localized TSC1/2 proteins function as Rheb GTPaseactivating proteins to repress mTOR signaling in response to the ROS generated within peroxisomes (Zhang et al., 2013). It is unknown how mTOR signaling is coordinated in response to nutrients and oxidative stresses. The PO-Lyso-MCS enriched with Rheb proteins may fulfill such a role, similar to that proposed for the lysosome-Golgi-MCS in the activation of mTOR signaling (Hao et al., 2018). However, TSC1/2 proteins were not detected in several proteomic studies of purified peroxisomes (Yifrach et al., 2018), and they dissociate from the lysosomes to the cytosol upon insulin stimulation, resulting in Rheb-mediated mTORC1 activation at lysosomes (Menon et al., 2014). These findings do not support the role of PO-Lyso-MCS in modulating mTORC1 signaling, as hypothesized above.

\section{PO-MCSS WITH OTHER ORGANELLES}

In addition, PO-MCSs with other organelles have been indicated. Peroxisomes move to the cell periphery near the PM by ACBD5 overexpression in neurons. This peroxisome redistribution does not depend on the interaction of ACBD5 with VAPs, suggesting that other ACBD5 interacting proteins may tether peroxisomes to PM (Wang et al., 2018). Live imaging of fusion cells expressing peroxisomal proteins fused to different fluorescence tags reveals that peroxisomes can interact with each other in a transient and long-term manner. The contact does not promote the exchange of matrix and membrane proteins, FAs, and $\mathrm{H}_{2} \mathrm{O}_{2}$ (Bonekamp et al., 2012). Global analysis of the organelle interactome reveals that peroxisomes contact the Golgi, in addition to the ER, mitochondria, LDs, and lysosomes (Valm et al., 2017). With more PO-contacting organelles being identified, their tethering components and functions remain to be characterized.

\section{DISCUSSION}

The MCSs open an avenue for peroxisomes to communicate with other organelles for diverse purposes, including organelle positioning, lipid supply for membrane elongation, and metabolism coordination, as discussed above. However, MCSs are much more complicated than initially thought. We highlight and discuss several features of PO-MCSs that may help future studies.

\section{Heterogeneity of Peroxisomes}

Peroxisomes are heterogeneous in terms of their number, morphology, distribution, and composition in different cells or environmental settings (Mast et al., 2020). For example, the number of peroxisomes increases when rodents are administered fibrate derivatives but decreases rapidly upon withdrawal of the drugs (Fahimi et al., 1982; Yokota, 1986; Yokota, 1993). Enveloped virus infection induces significant peroxisome biogenesis, promoting phospholipid plasmalogen synthesis for virus production (Jean Beltran et al., 2018). Peroxisomes also display heterogeneity in the same cell. An example is that two populations of peroxisomes that contain different ratios of lipid $\beta$-oxidation enzymes to catalase are isolated in HepG2 cells (Schrader et al., 1994). The population containing higher contents of $\beta$-oxidation enzymes may favor PO-MCSs for FA metabolism. In contrast, those with higher catalases may prefer MCSs for ROS crosstalk. Hence, PO-MCSs may differ in terms of their tethering mechanisms and functions under these varying conditions, and selecting an appropriate cell or animal model would facilitate PO-MCS studies.

\section{Unconventional PO-MCSs}

The identified PO-MCSs are tethered through different proteinprotein or protein-lipid complexes and manifest a variety of physiological roles. In addition, as each cellular organelle has a unique structure, the mode of PO-MCS formation may be organelle-specific, as discussed below.

Mitochondria are surrounded by double membranes, which separate the organelle into two compartments, the intermembrane space and the matrix. Peroxisomes may contact the outer membranes, as shown for the ER-Mito MCS (Murley and Nunnari, 2016). This MCS may indirectly facilitate metabolic exchange with the matrix through transporters located at the inner membranes. Alternatively, it is tempting to speculate that the PO-tethering complexes may extend into the inner membranes to facilitate the exchange of metabolites.

Compared to bilayer-bound organelles, LDs have phospholipid monolayer membranes that surround the lipid core. This unique monolayer of LDs may be continuous with the outer leaflet of the bilayer of apposing organelles, as has been shown for ER-LD-MCS (Wang et al., 2016). This MCS does not exist between bilayer membranes and is distinct from the traditional MCS. In yeast, PO-LD-MCS formation increases in peroxisome-inducing conditions. The MCSs are enriched in $\beta$-oxidation enzymes, suggesting their roles in FA transfer from LDs to peroxisomes. At these MCSs, peroxisomal protrusions extend into the core of LDs, likely representing the "bridging contact" (Binns et al., 2006). It remains to be investigated whether peroxisomes form such a "bridging contact" with LDs in mammalian cells.

\section{The Components at the PO-MCSs}

The identified PO-MCSs are tethered through the proteinprotein or protein-lipid complexes present on the opposing membranes of the organelles. The tethering complexes may play additional roles in the MCSs. For example, the tether M1 
Spastin also recruits membrane curvature-shaping proteins to promote lipid transfer at the PO-LD-MCS (Chang et al., 2019). The identified peroxisomal tethers play MCS-independent roles, such as in metabolic/enzymatic activities. Hence, to elucidate whether the phenotype is solely caused by defective PO-MCS formation, it is necessary to test if a mutant defective in its tethering activity but maintaining other activities inhibits POMCS functions. Multiple tethering pairs may also exist for the PO-MCS, as shown for the PO-ER-MCSs as discussed above and for other organelle MCS (Scorrano et al., 2019). In this scenario, loss of the identified PO-MCS tether does not necessarily reduce their contacts and/or functions because of the redundancy of the tethering complexes. In addition, other effector proteins may be enriched at the MCSs to regulate MCS formation or facilitate the exchange of metabolites (Scorrano et al., 2019). As discussed above, ATGL can be grouped as the effector protein that promotes lipolysis at the PO-LD-MCS during fasting (Kong et al., 2020).

Although some components of the PO-MCS have been elucidated as discussed above, many questions remain to be answered. Elucidating components, such as tethering complexes and effector proteins, at PO-MCSs is a major challenge but will profoundly increase our understanding of PO-MCSs.

\section{Coordination of PO-MCSs With Multiple Organelles}

Membrane contact sites do not function alone. MCSs must be integrated and coordinated in response to the changing cellular environment. Peroxisomes can contact multiple organelles simultaneously, as shown for the PO-interacting network in COS-7 cells (Valm et al., 2017). The peroxisome-contacting organelles can be shifted to other organelles in varying conditions. An example is that PO-Mito contact increases when MAM (mitochondrial associated membrane, a specialized ER subdomain)-mitochondria contacts are disrupted, resulting in a concomitant increase in IFN- $\beta$ signaling during virus infection (Horner et al., 2011). We discuss three potential mechanisms for coordinating PO-MCSs with multiple organelles as follows.

A study investigated the assembly of ER-MCSs with a variety of organelles through lipid-based phase separation (King et al., 2020). By employing hypotonic cell swelling, the ER and other membrane-bound organelles can be converted into micrometerscale large intracellular vesicles (LICVs). Upon cooling, the ERderived LICVs phase-partition into the ER ordered $\left(\mathrm{ER}_{o}\right)$ and disordered $\left(\mathrm{ER}_{d}\right)$ lipid domains. Interestingly, they find that the PO-ER-MCS is located at the $\mathrm{ER}_{o} / \mathrm{ER}_{d}$ interphase, involving association with a mitochondrion. A yeast study also found that peroxisomes contact the ER and mitochondria at the three-way junction through Pex11 interacting with Mdm34, a component of ERMES (ER-mitochondria encounter structure) (Mattiazzi Usaj et al., 2015). As the three organelles are crucial for lipid and redox metabolism, the MCS at the tri-junction interface may allow efficient transfer of these metabolites among them. Hence, establishing PO-MCSs at the junction interface may coordinate peroxisome crosstalk with multiple organelles to meet metabolic needs.
Membrane contact site coordination can be achieved through the regulation of common tethering molecules (Harper et al., 2020). For example, in yeast, the tethering proteins Lam6 and Vps13 proteins are present at multiple contact sites, and they shift from one MCS to the other in response to changing carbon sources (Elbaz-Alon et al., 2015; Lang et al., 2015). As discussed above, The ER-localized VAPA/B proteins act as tethers with many other organelles, including peroxisomes (Eisenberg-Bord et al., 2016) and the $\mathrm{PI}(4,5) \mathrm{P}_{2}$ lipid at peroxisomal membranes tethers peroxisomes to both lysosomes and the ER. Regulation of these common PO tethers, such as VAPA/B and $\mathrm{PI}(4,5) \mathrm{P}_{2}$, may provide mechanistic hints for PO-MCS coordination.

The movement of peroxisomes in mammalian cells preferentially depends on the microtubular network and allows peroxisomes to be uniformly dispersed for metabolism (Neuhaus et al., 2016). PO-LD-MCS depends on the motility of peroxisomes along microtubules through the kinesin-like motor KifC3 to promote lipolysis under fasting stress conditions (Kong et al., 2020). Microtubule disruption by nocodazole treatment in COS-7 cells decreases peroxisome contacts with all the examined organelles (the ER, mitochondria, LDs, Golgi, and lysosomes) (Valm et al., 2017). These studies suggest that organelle motility is critical for PO-MCS formation. In turn, the MCSs regulate the movement and position of peroxisomes, exemplified by the role of PO-ER-MCS in restricting peroxisome motility, as discussed above. Peroxisomes can hitchhike on the microtubule-based motor machinery of endosomes for long-range movements through PO-endosome-MCSs in the filamentous fungus Aspergillus nidulans. Given the crucial role of microtubules in organizing a variety of organelle contacts and the reciprocal relationships between organelle motility and contact (de Forges et al., 2012; Valm et al., 2017), it is tempting to speculate that modulating the microtubular network can serve as another strategy for PO-MCS coordination.

\section{CONCLUDING REMARKS}

In summary, the vital importance of peroxisomes is underscored not only by the essential metabolism within this organelle but also by the intimate crosstalk with other organelles. MCS formation is an efficient means by which peroxisomes communicate with other organelles. Some of the tethering mechanisms and physiological functions have been elucidated or suggested. These studies have increased our understanding of not only peroxisomes but also other organelles, including their biogenesis/turnover and metabolic functions. However, these studies are still in the early phase, and much that is unknown remains to be addressed. Future PO-MCS studies would shed novel insights into human diseases and determine their therapeutic targets.

\section{AUTHOR CONTRIBUTIONS}

CC, JL, and XQ wrote the draft of the manuscript. WW drew the figure and organized and proofread the manuscript. All authors contributed to the article and approved the submitted version. 


\section{FUNDING}

This work was supported by the National Natural Science Foundation of China (Nos. 81903016 and 81904024).

\section{REFERENCES}

Abu-Safieh, L., Alrashed, M., Anazi, S., Alkuraya, H., Khan, A. O., Hashem, M., et al. (2013). Autozygome-guided exome sequencing in retinal dystrophy patients reveals pathogenetic mutations and novel candidate disease genes. Genome Res. 23, 236-247. doi: 10.1101/gr.144105.112

Antonenkov, V. D., and Hiltunen, J. K. (2012). Transfer of metabolites across the peroxisomal membrane. Biochim. Biophys. Acta 1822, 1374-1386. doi: 10.1016/j.bbadis.2011.12.011

Baes, M., Gressens, P., Baumgart, E., Carmeliet, P., Casteels, M., Fransen, M., et al. (1997). A mouse model for Zellweger syndrome. Nat. Genet. 17, 49-57.

Bartz, R., Li, W. H., Venables, B., Zehmer, J. K., Roth, M. R., Welti, R., et al. (2007). Lipidomics reveals that adiposomes store ether lipids and mediate phospholipid traffic. J. Lipid Res. 48, 837-847. doi: 10.1194/jlr.m600413-jlr200

Baumgart, E., Vanhorebeek, I., Grabenbauer, M., Borgers, M., Declercq, P. E., Fahimi, H. D., et al. (2001). Mitochondrial alterations caused by defective peroxisomal biogenesis in a mouse model for Zellweger syndrome (PEX5 knockout mouse). Am. J. Pathol. 159, 1477-1494. doi: 10.1016/s0002-9440(10) 62534- 5

Binns, D., Januszewski, T., Chen, Y., Hill, J., Markin, V. S., and Zhao, Y. (2006). An intimate collaboration between peroxisomes and lipid bodies. J. Cell Biol. 173, 719-731. doi: $10.1083 /$ jcb.200511125

Blackstone, C. (2018). Converging cellular themes for the hereditary spastic paraplegias. Curr. Opin. Neurobiol. 51, 139-146. doi: 10.1016/j.conb.2018.04. 025

Bonekamp, N. A., Sampaio, P., Luers, G. H., and Schrader, M. (2012). Transient complex interactions of mammalian peroxisomes without exchange of matrix or membrane marker proteins. Traffic 13, 960-978. doi: 10.1111/j.1600-0854. 2012.01356.x

Booth, D. M., Enyedi, B., Geiszt, M., Varnai, P., and Hajnoczky, G. (2016). Redox nanodomains are induced by and control calcium signaling at the ERmitochondrial interface. Mol. Cell 63, 240-248. doi: 10.1016/j.molcel.2016.05. 040

Castro, I. G., Schuldiner, M., and Zalckvar, E. (2018). Mind the organelle gap peroxisome contact sites in disease. Trends Biochem. Sci. 43, 199-210. doi: 10.1016/j.tibs.2018.01.001

Chang, C. L., Weigel, A. V., Ioannou, M. S., Pasolli, H. A., Xu, C. S., and Peale, D. R. (2019). Spastin tethers lipid droplets to peroxisomes and directs fatty acid trafficking through ESCRT-III. J. Cell Biol. 218, 2583-2599. doi: 10.1083/jcb. 201902061

Chang, T. Y., Chang, C. C., Ohgami, N., and Yamauchi, Y. (2006). Cholesterol sensing, trafficking, and esterification. Annu. Rev. Cell Dev. Biol. 22, 129-157. doi: 10.1146/annurev.cellbio.22.010305.104656

Chu, B. B., Liao, Y. C., Qi, W., Xie, C., Du, X., Wang, J., et al. (2015). Cholesterol transport through lysosome-peroxisome membrane contacts. Cell 161, 291306. doi: 10.1016/j.cell.2015.02.019

Cook, K. C., Moreno, J. A., Jean Beltran, P. M., and Cristea, I. M. (2019). Peroxisome plasticity at the virus-host interface. Trends Microbiol. 27, 906-914. doi: 10.1016/j.tim.2019.06.006

Costello, J. L., Castro, I. G., Hacker, C., Schrader, T. A., Metz, J., Zeuschner, D., et al. (2017a). ACBD5 and VAPB mediate membrane associations between peroxisomes and the ER. J. Cell Biol. 216, 331-342. doi: 10.1083/jcb.201607055

Costello, J. L., Castro, I. G., Schrader, T. A., Islinger, M., and Schrader, M. (2017b). Peroxisomal ACBD4 interacts with VAPB and promotes ER-peroxisome associations. Cell Cycle 16, 1039-1045. doi: 10.1080/15384101.2017.1314422

de Forges, H., Bouissou, A., and Perez, F. (2012). Interplay between microtubule dynamics and intracellular organization. Int. J. Biochem. Cell Biol. 44, 266-274. doi: 10.1016/j.biocel.2011.11.009

Delille, H. K., Alves, R., and Schrader, M. (2009). Biogenesis of peroxisomes and mitochondria: linked by division. Histochem. Cell Biol. 131, 441-446. doi: 10.1007/s00418-009-0561-9

\section{ACKNOWLEDGMENTS}

The authors apologize for any citations absent due to space constraints.

Dirkx, R., Vanhorebeek, I., Martens, K., Schad, A., Grabenbauer, M., Fahimi, D., et al. (2005). Absence of peroxisomes in mouse hepatocytes causes mitochondrial and ER abnormalities. Hepatology 41, 868-878. doi: 10.1002/ hep. 20628

Eisenberg-Bord, M., Shai, N., Schuldiner, M., and Bohnert, M. (2016). A tether is a tether is a tether: tethering at membrane contact sites. Dev. Cell 39, 395-409. doi: 10.1016/j.devcel.2016.10.022

Elbaz-Alon, Y., Eisenberg-Bord, M., Shinder, V., Stiller, S. B., Shimoni, E., Wiedemann, N., et al. (2015). Lam6 regulates the extent of contacts between organelles. Cell Rep. 12, 7-14. doi: 10.1016/j.celrep.2015.06.022

Engelen, M., Kemp, S., de Visser, M., van Geel, B. M., Wanders, R. J., and Aubourg, P. (2012). X-linked adrenoleukodystrophy (X-ALD): clinical presentation and guidelines for diagnosis, follow-up and management. Orphanet J. Rare Dis. 7:51. doi: 10.1186/1750-1172-7-51

Errico, A., Ballabio, A., and Rugarli, E. I. (2002). Spastin, the protein mutated in autosomal dominant hereditary spastic paraplegia, is involved in microtubule dynamics. Hum. Mol. Genet. 11, 153-163. doi: 10.1093/hmg/11.2.153

Fahimi, H. D., Reinicke, A., Sujatta, M., Yokota, S., Ozel, M., Hartig, F., et al. (1982). The short- and long-term effects of bezafibrate in the rat. Ann. N. Y. Acad. Sci. 386, 111-135. doi: 10.1111/j.1749-6632.1982.tb21410.x

Fan, J., Li, X., Issop, L., Culty, M., and Papadopoulos, V. (2016). ACBD2/ECI2mediated peroxisome-mitochondria interactions in leydig cell steroid biosynthesis. Mol. Endocrinol. 30, 763-782. doi: 10.1210/me.2016-1008

Ferdinandusse, S., Falkenberg, K. D., Koster, J., Mooyer, P. A., Jones, R., and Waterham, H. R. (2017). ACBD5 deficiency causes a defect in peroxisomal very long-chain fatty acid metabolism. J. Med. Genet. 54, 330-337. doi: 10.1136/ jmedgenet-2016-104132

Fransen, M., and Lismont, C. (2018). Peroxisomes and cellular oxidant/antioxidant balance: protein redox modifications and impact on inter-organelle communication. Subcell. Biochem. 89, 435-461. doi: 10.1007/978-98113-2233-4_19

Friedman, J. R., Lackner, L. L., West, M., DiBenedetto, J. R., Nunnari, J., and Voeltz, G. K. (2011). ER tubules mark sites of mitochondrial division. Science 334, 358-362. doi: 10.1126/science. 1207385

Hao, F., Kondo, K., Itoh, T., Ikari, S., Nada, S., Okada, M., et al. (2018). Rheb localized on the Golgi membrane activates lysosome-localized mTORC1 at the Golgi-lysosome contact site. J. Cell Sci. 131:jcs208017.

Hardeman, D., Zomer, H. W., Schutgens, R. B., Tager, J. M., and van den Bosch, H. (1990). Effect of peroxisome proliferation on ether phospholipid biosynthesizing enzymes in rat liver. Int. J. Biochem. 22, 1413-1418. doi: 10. 1016/0020-711x(90)90231-q

Harper, C. S., White, A. J., and Lackner, L. L. (2020). The multifunctional nature of mitochondrial contact site proteins. Curr. Opin. Cell Biol. 65, 58-65. doi: 10.1016/j.ceb.2020.02.010

Haze, K., Yoshida, H., Yanagi, H., Yura, T., and Mori, K. (1999). Mammalian transcription factor ATF6 is synthesized as a transmembrane protein and activated by proteolysis in response to endoplasmic reticulum stress. Mol. Biol. Cell 10, 3787-3799. doi: 10.1091/mbc.10.11.3787

Hicks, L., and Fahimi, H. D. (1977). Peroxisomes (microbodies) in the myocardium of rodents and primates. A comparative Ultrastructural cytochemical study. Cell Tissue Res. 175, 467-481.

Horner, S. M., Liu, H. M., Park, H. S., Briley, J., and Gale, M. Jr. (2011) Mitochondrial-associated endoplasmic reticulum membranes (MAM) form innate immune synapses and are targeted by hepatitis C virus. Proc. Natl. Acad. Sci. U.S.A. 108, 14590-14595. doi: 10.1073/pnas.1110133108

Hu, A., Zhao, X. T., Tu, H., Xiao, T., Fu, T., Wang, Y., et al. (2018). PIP4K2A regulates intracellular cholesterol transport through modulating $\mathrm{PI}(4,5) \mathrm{P} 2$ homeostasis. J. Lipid Res. 59, 507-514. doi: 10.1194/jlr.m082149

Hua, R., Cheng, D., Coyaud, E., Freeman, S., Wang, Y., Vissa, A., et al. (2017) VAPs and ACBD5 tether peroxisomes to the ER for peroxisome maintenance and lipid homeostasis. J. Cell Biol. 216, 367-377. doi: 10.1083/jcb.201608128 
Jean Beltran, P. M., Cook, K. C., Hashimoto, Y., Galitzine, C., Murray, L. A., Vitek, O., et al. (2018). Infection-induced peroxisome biogenesis is a metabolic strategy for Herpesvirus replication. Cell Host Microbe 24, 526-541.e7. doi: 10.1016/j.chom.2018.09.002

Kagan, J. C. (2012). Signaling organelles of the innate immune system. Cell 151, 1168-1178. doi: 10.1016/j.cell.2012.11.011

Kamemura, K., and Chihara, T. (2019). Multiple functions of the ER-resident VAP and its extracellular role in neural development and disease. J. Biochem. 165, 391-400. doi: 10.1093/jb/mvz011

Kim, S., Leal, S. S., Ben Halevy, D., Gomes, C. M., and Lev, S. (2010). Structural requirements for VAP-B oligomerization and their implication in amyotrophic lateral sclerosis-associated VAP-B(P56S) neurotoxicity. J. Biol. Chem. 285, 13839-13849. doi: 10.1074/jbc.m109.097345

King, C., Sengupta, P., Seo, A. Y., and Lippincott-Schwartz, J. (2020). ER membranes exhibit phase behavior at sites of organelle contact. Proc. Natl. Acad. Sci. U.S.A. 117, 7225-7235. doi: 10.1073/pnas.1910854117

Knoblach, B., Sun, X., Coquelle, N., Fagarasanu, A., Poirier, R. L., and Rachubinski, R. A. (2013). An ER-peroxisome tether exerts peroxisome population control in yeast. EMBO J. 32, 2439-2453. doi: 10.1038/emboj.2013.170

Kong, J., Ji, Y., Jeon, Y. G., Han, J. S., Han, K. H., Lee, J. H., et al. (2020). Spatiotemporal contact between peroxisomes and lipid droplets regulates fasting-induced lipolysis via PEX5. Nat. Commun. 11:578.

Kustatscher, G., Grabowski, P., Schrader, T. A., Passmore, J. B., Schrader, M., and Rappsilber, J. (2019). Co-regulation map of the human proteome enables identification of protein functions. Nat. Biotechnol. 37, 1361-1371. doi: 10. 1038/s41587-019-0298-5

Lang, A. B., John Peter, A. T., Walter, P., and Kornmann, B. (2015). ERmitochondrial junctions can be bypassed by dominant mutations in the endosomal protein Vps13. J. Cell Biol. 210, 883-890. doi: 10.1083/jcb. 201502105

Lange, Y., Swaisgood, M. H., Ramos, B. V., and Steck, T. L. (1989). Plasma membranes contain half the phospholipid and $90 \%$ of the cholesterol and sphingomyelin in cultured human fibroblasts. J. Biol. Chem. 264, 3786-3793.

Lev, S., Ben Halevy, D., Peretti, D., and Dahan, N. (2008). The VAP protein family: from cellular functions to motor neuron disease. Trends Cell Biol. 18, 282-290. doi: $10.1016 /$ j.tcb.2008.03.006

Lopez-Erauskin, J., Galino, J., Ruiz, M., Cuezva, J. M., Fabregat, I., Cacabelos, D., et al. (2013). Impaired mitochondrial oxidative phosphorylation in the peroxisomal disease X-linked adrenoleukodystrophy. Hum. Mol. Genet. 22, 3296-3305. doi: 10.1093/hmg/ddt186

Mast, F. D., Rachubinski, R. A., and Aitchison, J. D. (2020). Peroxisome prognostications: exploring the birth, life, and death of an organelle. J. Cell Biol. 219:e201912100.

Mattiazzi Usaj, M., Brloznik, M., Kaferle, P., Zitnik, M., Wolinski, H., Leitner, F., et al. (2015). Genome-wide localization study of yeast Pex11 identifies peroxisome-mitochondria interactions through the ERMES complex. J. Mol. Biol. 427, 2072-2087. doi: 10.1016/j.jmb.2015.03.004

Menon, S., Dibble, C. C., Talbott, G., Hoxhaj, G., Valvezan, A. J., Takahashi, H., et al. (2014). Spatial control of the TSC complex integrates insulin and nutrient regulation of mTORC1 at the lysosome. Cell 156, 771-785. doi: 10.1016/j.cell. 2013.11.049

Munn, N. J., Arnio, E., Liu, D., Zoeller, R. A., and Liscum, L. (2003). Deficiency in ethanolamine plasmalogen leads to altered cholesterol transport. J. Lipid Res. 44, 182-192. doi: 10.1194/jlr.m200363-jlr200

Murley, A., and Nunnari, J. (2016). The emerging network of mitochondriaorganelle contacts. Mol. Cell 61, 648-653. doi: 10.1016/j.molcel.2016.01.031

Nazarko, T. Y., Ozeki, K., Till, A., Ramakrishnan, G., Lotfi, P., Yan, M., et al. (2014). Peroxisomal Atg37 binds Atg30 or palmitoyl-CoA to regulate phagophore formation during pexophagy. J. Cell Biol. 204, 541-557. doi: 10.1083/jcb. 201307050

Neuhaus, A., Eggeling, C., Erdmann, R., and Schliebs, W. (2016). Why do peroxisomes associate with the cytoskeleton? Biochim. Biophys. Acta 1863, 1019-1026. doi: 10.1016/j.bbamcr.2015.11.022

Novikoff, P. M., and Novikoff, A. B. (1972). Peroxisomes in absorptive cells of mammalian small intestine. J. Cell Biol. 53, 532-560. doi: 10.1083/jcb.53.2.532

Prinz, W. A. (2014). Bridging the gap: membrane contact sites in signaling, metabolism, and organelle dynamics. J. Cell Biol. 205, 759-769. doi: 10.1083/ jcb.201401126
Prinz, W. A., Toulmay, A., and Balla, T. (2020). The functional universe of membrane contact sites. Nat. Rev. Mol. Cell Biol. 21, 7-24. doi: 10.1038/s41580019-0180-9

Rowland, A. A., Chitwood, P. J., Phillips, M. J., and Voeltz, G. K. (2014). ER contact sites define the position and timing of endosome fission. Cell 159, 1027-1041. doi: 10.1016/j.cell.2014.10.023

Sargsyan, Y., and Thoms, S. (2020). Staying in healthy contact: how peroxisomes interact with other cell organelles. Trends Mol. Med. 26, 201-214. doi: 10.1016/ j.molmed.2019.09.012

Saxton, R. A., and Sabatini, D. M. (2017). mTOR signaling in growth. Metab. Dis. Cell 169, 361-371.

Schaumburg, H. H., Richardson, E. P., Johnson, P. C., Cohen, R. B., Powers, J. M., and Raine, C. S. (1972). Schilder's disease. Sex-linked recessive transmission with specific adrenal changes. Arch. Neurol. 27, 458-460.

Schrader, M., Baumgart, E., Volkl, A., and Fahimi, H. D. (1994). Heterogeneity of peroxisomes in human hepatoblastoma cell line HepG2. Evidence of distinct subpopulations. Eur. J. Cell Biol. 64, 281-294.

Schrader, M., Bonekamp, N. A., and Islinger, M. (2012). Fission and proliferation of peroxisomes. Biochim. Biophys. Acta 1822, 1343-1357. doi: 10.1016/j.bbadis. 2011.12.014

Schrader, M., Grille, S., Fahimi, H. D., and Islinger, M. (2013). Peroxisome interactions and cross-talk with other subcellular compartments in animal cells. Subcell. Biochem. 69, 1-22. doi: 10.1007/978-94-007-6889-5_1

Schrader, M., Kamoshita, M., and Islinger, M. (2020). Organelle interplayperoxisome interactions in health and disease. J. Inherit. Metab. Dis. 43, 71-89. doi: 10.1002/jimd.12083

Scorrano, L., Emr, S., Giordano, F., Hajnoczky, G., Kornmann, B., and Lackner, L. L. (2019). Coming together to define membrane contact sites. Nat. Commun. 10:1287.

Shai, N., Schuldiner, M., and Zalckvar, E. (2016). No peroxisome is an island Peroxisome contact sites. Biochim. Biophys. Acta 1863, 1061-1069. doi: 10. 1016/j.bbamcr.2015.09.016

Shai, N., Yifrach, E., Cohen, N., Bibi, C., Meurisse, J., Schuster, R., et al. (2018). Systematic mapping of contact sites reveals tethers and a function for the peroxisome-mitochondria contact. Nat. Commun. 9:1761.

Smith, J. J., and Aitchison, J. D. (2013). Peroxisomes take shape. Nat. Rev. Mol. Cell Biol. 14, 803-817. doi: 10.1038/nrm3700

Sugiura, A., Mattie, S., Prudent, J., and McBride, H. M. (2017). Newly born peroxisomes are a hybrid of mitochondrial and ER-derived pre-peroxisomes. Nature 542, 251-254. doi: 10.1038/nature21375

Sugiura, A., McLelland, G. L., Fon, E. A., and McBride, H. M. (2014). A new pathway for mitochondrial quality control: mitochondrial-derived vesicles. EMBO J. 33, 2142-2156. doi: 10.15252/embj.201488104

Suzuki, Y., Shimozawa, N., Orii, T., Tsukamoto, T., Osumi, T., Fujiki, Y., et al. (2001). Genetic and molecular bases of peroxisome biogenesis disorders. Genet. Med. 3, 372-376. doi: 10.1097/00125817-200109000-00007

Thai, T. P., Rodemer, C., Jauch, A., Hunziker, A., Moser, A., Gorgas, K., et al. (2001). Impaired membrane traffic in defective ether lipid biosynthesis. Hum. Mol. Genet. 10, 127-136. doi: 10.1093/hmg/10.2.127

Thiam, A. R., and Dugail, I. (2019). Lipid droplet-membrane contact sites - from protein binding to function. J. Cell Sci. 132:jcs230169. doi: 10.1242/jcs.230169

Torres, S. E., Gallagher, C. M., Plate, L., Gupta, M., Liem, C. R., Guo, X., et al. (2019). Ceapins block the unfolded protein response sensor ATF6alpha by inducing a neomorphic inter-organelle tether. eLife 8:e46595.

Valm, A. M., Cohen, S., Legant, W. R., Melunis, J., Hershberg, U., Wait, E., et al. (2017). Applying systems-level spectral imaging and analysis to reveal the organelle interactome. Nature 546, 162-167. doi: 10.1038/nature 22369

Verfaillie, T., Rubio, N., Garg, A. D., Bultynck, G., Rizzuto, R., Decuypere, J. P., et al. (2012). PERK is required at the ER-mitochondrial contact sites to convey apoptosis after ROS-based ER stress. Cell Death Differ. 19, 1880-1891. doi: $10.1038 / \mathrm{cdd} .2012 .74$

Walbrecq, G., Wang, B., Becker, S., Hannotiau, A., Fransen, M., and Knoops, B. (2015). Antioxidant cytoprotection by peroxisomal peroxiredoxin-5. Free Radic. Biol. Med. 84, 215-226. doi: 10.1016/j.freeradbiomed.2015.02.032

Walton, P. A., and Pizzitelli, M. (2012). Effects of peroxisomal catalase inhibition on mitochondrial function. Front. Physiol. 3:108. doi: 10.3389/fphys.2012. 00108 
Wang, B., Brees, C., Rubio, N., Nordgren, M., Apanasets, O., Kunze, M., et al. (2013). Mitochondria are targets for peroxisome-derived oxidative stress in cultured mammalian cells. Free Radic. Biol. Med. 65, 882-894. doi: 10.1016/ j.freeradbiomed.2013.08.173

Wang, H., Becuwe, M., Housden, B. E., Chitraju, C., Porras, A. J., and Graham, M. M. (2016). Seipin is required for converting nascent to mature lipid droplets. eLife 5:e16582.

Wang, Y., Metz, J., Costello, J. L., Passmore, J., Schrader, M., Schultz, C., et al. (2018). Intracellular redistribution of neuronal peroxisomes in response to ACBD5 expression. PLoS One 13:e0209507. doi: 10.1371/journal.pone.0209507

Waterham, H. R., Ferdinandusse, S., and Wanders, R. J. (2016). Human disorders of peroxisome metabolism and biogenesis. Biochim. Biophys. Acta 1863, 922933. doi: 10.1016/j.bbamcr.2015.11.015

Welte, M. A. (2015). Expanding roles for lipid droplets. Curr. Biol. 25, R470-R481.

Xiao, J., Luo, J., Hu, A., Xiao, T., Li, M., Kong, Z., et al. (2019). Cholesterol transport through the peroxisome-ER membrane contacts tethered by $\mathrm{PI}(4,5) \mathrm{P} 2$ and extended synaptotagmins. Sci. China Life Sci. 62, 1117-1135. doi: 10.1007/ s11427-019-9569-9

Yagita, Y., Shinohara, K., Abe, Y., Nakagawa, K., Alkuraya, F. S., and Fujiki, Y. (2017). Deficiency of a retinal dystrophy protein, Acyl-CoA binding domain-containing 5 (ACBD5), impairs peroxisomal beta-oxidation of verylong-chain fatty acids. J. Biol. Chem. 292, 691-705. doi: 10.1074/jbc.m116. 760090

Yifrach, E., Fischer, S., Oeljeklaus, S., Schuldiner, M., Zalckvar, E., and Warscheid, B. (2018). Defining the mammalian peroxisomal proteome. Subcell. Biochem. 89, 47-66. doi: 10.1007/978-981-13-2233-4_2
Yokota, S. (1986). Quantitative immunocytochemical studies on differential induction of serine:pyruvate aminotransferase in mitochondria and peroxisomes of rat liver cells by administration of glucagon or di(2-ethylhexyl)phthalate. Histochemistry 85, 145-155. doi: 10.1007/ bf00491762

Yokota, S. (1993). Formation of autophagosomes during degradation of excess peroxisomes induced by administration of dioctyl phthalate. Eur. J. Cell Biol. 61, 67-80.

Zhang, J., Kim, J., Alexander, A., Cai, S., Tripathi, D. N., and Dere, R. (2013). A tuberous sclerosis complex signalling node at the peroxisome regulates mTORC1 and autophagy in response to ROS. Nat. Cell Biol. 15, 1186-1196. doi: $10.1038 / \mathrm{ncb} 2822$

Zhang, J., Tripathi, D. N., Jing, J., Alexander, A., Kim, J., and Powell, R. T. (2015). ATM functions at the peroxisome to induce pexophagy in response to ROS. Nat. Cell Biol. 17, 1259-1269. doi: 10.1038/ncb3230

Conflict of Interest: The authors declare that the research was conducted in the absence of any commercial or financial relationships that could be construed as a potential conflict of interest.

Copyright (c) 2020 Chen, Li, Qin and Wang. This is an open-access article distributed under the terms of the Creative Commons Attribution License (CC BY). The use, distribution or reproduction in other forums is permitted, provided the original author(s) and the copyright owner(s) are credited and that the original publication in this journal is cited, in accordance with accepted academic practice. No use, distribution or reproduction is permitted which does not comply with these terms. 